CERN-PH-TH/2006-079

\title{
A possible symmetry of the $\nu \mathrm{MSM}$
}

\author{
Mikhail Shaposhnikov ${ }^{1,2}$ \\ 1 Institut de Théorie des Phénomènes Physiques, \\ Ecole Polytechnique Fédérale de Lausanne, CH-1015 Lausanne, Switzerland \\ ${ }^{2}$ Theory Division, CERN, CH-1211 Geneve 23, Switzerland
}

(Dated: October 31, 2006)

\begin{abstract}
To explain the dark matter and the baryon asymmetry of the Universe, the parameters of the $\nu$ MSM (an extension of the Minimal Standard Model by three singlet neutrinos with masses smaller than the electroweak scale) must be fine-tuned: one of the masses should be in the $\mathcal{O}(10)$ keV region to provide a candidate for the dark-matter particle, while two other masses must be almost the same to enhance the CP-violating effects in the sterile neutrino oscillations leading to the baryon asymmetry. We show that a specifically defined global lepton-number symmetry, broken on the level of $\mathcal{O}\left(10^{-4}\right)$ leads to the required pattern of sterile neutrino masses being consistent with the data on neutrino oscillations. Moreover, the existence of this symmetry allows to fix the flavour structure of couplings of singlet fermions to the particles of the Standard Model and indicates that their masses are likely to be smaller than $\mathcal{O}(1) \mathrm{GeV}$, opening a possibility of their search in decays of charmed, beauty and even $K$ or $\pi$-mesons.

PACS numbers: 14.60.Pq, 98.80.Cq, 95.35.+d
\end{abstract}




\section{INTRODUCTION}

There is compelling evidence that the Minimal Standard Model (MSM) of strong and electroweak interactions is not complete. There are several experimental and observational facts that cannot be explained by the MSM. These are neutrino oscillations, the presence of dark matter in the Universe, the baryon asymmetry of the Universe, its flatness, and the existence of cosmological perturbations necessary for structure formation. Indeed, in the MSM neutrinos are strictly massless and do not oscillate. The MSM does not have any candidate for non-baryonic dark matter. Moreover, with the present experimental limit on the Higgs mass, the high-temperature phase transition, required for electroweak baryogenesis, is absent. Finally, the couplings of the single scalar field of the MSM are too large for the Higgs boson to play the role of the inflaton.

In this paper we will assume that a simple renormalizable extension of the MSM by three singlet right-handed neutrinos and by a real scalar field (inflaton) with masses smaller than the electroweak scale is a correct effective theory up to some high-energy scale, which may be as large as the Planck scale [1, 2, 3]. Following [1, 2], we will call this model "the $\nu$ MSM", underlying the fact that it is the extension of the MSM in the neutrino sector. We will use the same name for a theory with inflaton [3]. Contrary to Grand Unified Theories, the $\nu \mathrm{MSM}$ does not have any internal hierarchy problem, simply because it is a theory with a single mass scale. Moreover, as the energy behaviour of the gauge couplings in this theory is the same as in the MSM, the absence of gauge-coupling unification in it indicates that there may be no grand unification, in accordance with our assumption of the validity of this theory up to the Planck scale. As well as the MSM, the $\nu$ MSM does not provide any explanation why the weak scale is much smaller than the Planck scale.

As we demonstrated in [1, 2], the $\nu$ MSM can explain simultaneously dark matter and baryon asymmetry of the Universe being consistent with neutrino masses and mixings observed experimentally ${ }^{1}$. Moreover, in [3] we have shown that inclusion of an inflaton with scale-invariant couplings to the fields of the $\nu \mathrm{MSM}$ allows us to have inflation and provides a common source for electroweak symmetry breaking and Majorana neutrino masses of singlet fermions - sterile neutrinos. The role of the dark matter is played by the lightest sterile

\footnotetext{
${ }^{1}$ We do not include here the LSND anomaly [4], which will be tested in MiniBooNE experiment [5].
} 
neutrino with mass $m_{s}$ in the keV range, which can be created in active-sterile neutrino oscillations [6] or through the coupling to the inflaton [3]. In the latter case, the dark matter sterile neutrino can satisfy [3, 7] all astrophysical and cosmological constraints on its mass and mixing angle with an active neutrino [8, 9, 10, 11, 12, 13, 14]. In addition, the coherent oscillations of two other, almost degenerate, sterile neutrinos lead to the creation of baryon asymmetry of the Universe [2] through the splitting of the lepton number between active and sterile neutrinos [15] and electroweak sphalerons [16]. For other astrophysical applications of sterile neutrinos see [17, 18, 19].

To explain simultaneously the dark matter and baryon asymmetry of the Universe, the parameters of the $\nu \mathrm{MSM}$ must be highly fine-tuned [1, 2]. If $m_{s}$, the mass of the lightest sterile neutrino $N_{1}$, playing the role of dark matter, is in the $\mathrm{keV}$ range (this mass interval is suggested, for example, by observations of dwarf galaxies [20] and by arguments coming from structure formation [21] ), it must be about five orders of magnitude smaller than the masses of the heavier sterile neutrinos, $N_{2}$ and $N_{3}$. The Yukawa coupling of $N_{1}$ to left-handed leptons $\left(f_{1} \lesssim 10^{-12}\right)$ must be at least five orders of magnitude smaller than the similar coupling of $N_{2}$ and $N_{3}$ (these constraint are coming from the Big Bang Nucleosynthesis $(\mathrm{BBN})$ ). Moreover, the heavier sterile neutrinos $N_{2}$ and $N_{3}$ must be degenerate in mass at least in one part in $10^{5}$. This looks rather bizarre. However, these fine-tunings can be taken as an indication of some new (slightly broken) symmetry, which the $\nu$ MSM must satisfy and which follows from some unknown underlying physics, related, perhaps, to the Planck scale. This paper is an attempt to reveal such a symmetry. We will show that the above-mentioned fine tunings can be explained by the approximate conservation of a specifically defined lepton number, explicitly broken by Majorana mass terms and Yukawa coupling constants.

We would like to stress that this work does not try to shed any light on the structure of the active neutrino mass matrix. For example, we have nothing to say about the smallness of the mixing angle $\theta_{13}$ or why the mixing angle $\theta_{23}$ is so close to maximal. Our aim is different: we want to understand, on the basis of the available experimental data on neutrino oscillations and on input from cosmology, described above, what are the properties of heavier singlet fermions, and, in particular, what are the values of their masses and couplings to left-handed leptons. We will show that if the breaking of the lepton number symmetry (that makes the heavier sterile neutrinos be degenerate and the dark matter neutrino be light) is tiny, then the masses of singlet fermions should be smaller than $1 \mathrm{GeV}$. Thus, these particles can be 
searched for in decays of charmed and beauty mesons, $\tau$-lepton and, possibly $K$ or $\pi$-mesons (in the latter case a number of experimental and BBN constraints already exist). In addition, we show that the ratios of the Yukawa couplings of sterile neutrinos to the Higgs and SM leptons can be found from the data on active neutrino mass matrix.

The paper is organized as follows. In Section 2 we will discuss the requisite symmetry of the $\nu$ MSM that can explain the above-mentioned fine tunings and construct a symmetric $\nu$ MSM. In the third Section we introduce the global symmetry breaking and analyse the active neutrino mixing matrix; in the fourth we will discuss the baryon asymmetry of the Universe and dark matter in the nearly symmetric $\nu$ MSM. In Section 5 we discuss the values of the masses of heavy neutral leptons and consider the Yukawa couplings of singlet fermions to the Higgs and left-handed leptons. We conclude in Section 6.

\section{THE SYMMETRIC $\nu$ MSM}

The Lagrangian of the $\nu \mathrm{MSM}$ is

$$
\mathcal{L}_{\nu \mathrm{MSM}}=\mathcal{L}_{\mathrm{MSM}}+\bar{N}_{I} i \partial_{\mu} \gamma^{\mu} N_{I}-F_{\alpha I} \bar{L}_{\alpha} N_{I} \Phi-\frac{M_{I J}}{2} \bar{N}_{I}^{c} N_{J}+\text { h.c. }
$$

where $\Phi$ and $L_{\alpha}(\alpha=e, \mu, \tau)$ are respectively the Higgs and lepton doublets, $F$ is a matrix of Yukawa coupling constants, and $M_{I}$ are the Majorana masses. By the field redefinitions, the Majorana mass matrix can be taken to be real and diagonal ${ }^{2}, M_{I J}=M_{I} \delta_{I J}$. Without loss of generality we choose the matrix of Yukawa couplings of left-handed doublets $L_{\alpha}$ and right-handed charged leptons $E_{\alpha}$ to be real and diagonal.

Suppose now that all fine tunings of the $\nu \mathrm{MSM}$, necessary for the production of dark matter and baryon asymmetry of the Universe, are exactly satisfied. (To distinguish the fields in this case from those appearing in (1), we will use a "tilde" in the notation.) Namely, let us require that $\widetilde{N}_{2}$ and $\widetilde{N}_{3}$ be exactly degenerate in mass, that $\widetilde{N}_{1}$ be exactly massless and not to interact at all with left-handed doublets. What kind of global symmetry can lead to this pattern automatically?

\footnotetext{
${ }^{2}$ In [3] the Majorana masses were coming from interaction with the inflaton field. In this paper we replace the inflaton field by its vacuum expectation value, since the discussion of symmetry properties does not require any analysis of inflaton dynamics.
} 
As for degeneracy between $\widetilde{N}_{2}$ and $\widetilde{N}_{3}$, the unique choice (others are equivalent to this one after linear transformations of fields) is the $\mathrm{U}(1)$ symmetry:

$$
\tilde{N}_{2} \rightarrow e^{-i \alpha_{L}} \tilde{N}_{2}, \quad \widetilde{N}_{3} \rightarrow e^{i \alpha_{L}} \tilde{N}_{3}
$$

whereas for $\widetilde{N}_{1}$ this is simply a chiral symmetry

$$
\widetilde{N}_{1} \rightarrow e^{i \beta_{L}} \widetilde{N}_{1}
$$

One can say that $\widetilde{N}_{2}$ and $\widetilde{N}_{3}$ form a Dirac spinor $\Psi=\widetilde{N}_{2}+\widetilde{N}_{3}^{c}$, or, in other words, $\widetilde{N}_{2}$ and $\widetilde{N}_{3}$ represent particle and antiparticle. As a result, the free Lagrangian for sterile neutrinos is

$$
L_{\text {free }}=\tilde{\widetilde{N}}_{I} i \partial_{\mu} \gamma^{\mu} \widetilde{N}_{I}-M \tilde{\bar{N}}_{2}^{c} \widetilde{N}_{3}
$$

It is invariant under $U(1)_{23} \times U(1)_{1}$ symmetry. One can equally say that there is just one $U(1)_{L}$ symmetry and that the quantum numbers of $\tilde{N}_{I}$ with respect to this symmetry are $(q,-1,1)$ for $\widetilde{N}_{1}, \widetilde{N}_{2}$ and $\widetilde{N}_{3}$ respectively, and that $q \neq 0$, since for $q=0$ a Majorana mass term for $\widetilde{N}_{1}$ is allowed. Though for $q= \pm 1$ the mass mixing terms between $\widetilde{N}_{2,3}$ and $\widetilde{N}_{1}$ are admitted, the theory still has one massless state in the sterile neutrino sector. We will call the quantum number associated with this $U(1)_{L}$ symmetry "the lepton number" for reasons that will become obvious below. The parameter $M$ is nothing but the mass of $\widetilde{N}_{2,3}$. In addition to $U(1)_{L}$ symmetry specified above the Lagrangian $\mathcal{L}_{\nu \mathrm{MSM}}+L_{\text {free }}$ is invariant under $U(1)_{e} \times U(1)_{\mu} \times U(1)_{\tau}$ symmetry corresponding to separate conservation of $e, \mu$ and $\tau$ leptonic numbers. At this stage the total global symmetry in the leptonic sector is $U(1)^{4}$.

Now, we would like to construct an interaction, consistent with our $U(1)_{L}$ symmetry, between $\widetilde{N}_{2,3}$ and $L_{\alpha}$, but forbid the interaction between $\widetilde{N}_{1}$ and $L_{\alpha}$. The assumption that all leptonic doublets are allowed to interact with sterile neutrinos $\widetilde{N}_{2,3}$ and with right-handed charged leptons leads us to four inequivalent charge assignments for active leptonic flavours: $(1,1,1),(1,1,-1),(1,-1,1)$ and $(-1,1,1)$. Then, the interactions between $\widetilde{N}_{1}$ and $L_{\alpha}$ are not allowed for $q \neq \pm 1$. So, we have to consider four different Yukawa matrices $F_{0}$ :

$$
\text { Model I : } \quad \mathrm{F}_{0}=\left(\begin{array}{ccc}
0 & h_{12} & 0 \\
0 & h_{22} & 0 \\
0 & h_{32} & 0
\end{array}\right) \text {, Model II : } \quad \mathrm{F}_{0}=\left(\begin{array}{ccc}
0 & 0 & h_{13} \\
0 & 0 & h_{23} \\
0 & h_{32} & 0
\end{array}\right) \text {, }
$$




$$
\text { Model III : } \quad \mathrm{F}_{0}=\left(\begin{array}{ccc}
0 & 0 & h_{13} \\
0 & h_{22} & 0 \\
0 & 0 & h_{33}
\end{array}\right) \text {, Model IV : } \quad \mathrm{F}_{0}=\left(\begin{array}{ccc}
0 & h_{12} & 0 \\
0 & 0 & h_{23} \\
0 & 0 & h_{33}
\end{array}\right) \text {. }
$$

where $h_{i j}$ can be taken to be real. In any of these models the total global symmetry in the leptonic sector is broken explicitly down to $U(1)_{L}$.

To summarize, we achieved the initial goal: the theory is invariant under $U(1)_{L}$ global symmetry, which guarantees the degeneracy between $\widetilde{N}_{2}$ and $\widetilde{N}_{3}$, the absence of mass for $\widetilde{N}_{1}$, and the absence of interactions between $\widetilde{N}_{1}$ and $L_{\alpha}$.

To choose the charge assignment that could best fit the data on neutrino oscillations, let us consider the active neutrino mass matrix, which we define following ref. [22]:

$$
M_{\nu}=V^{*} \cdot \operatorname{diag}\left(m_{1}, m_{2} e^{2 i \delta_{1}}, m_{3} e^{2 i \delta_{2}}\right) \cdot V^{\dagger},
$$

with $V=R\left(\theta_{23}\right) \operatorname{diag}\left(1, \mathrm{e}^{\mathrm{i} \delta_{3}}, 1\right) \mathrm{R}\left(\theta_{13}\right) \mathrm{R}\left(\theta_{12}\right)$ the active neutrino mixing matrix [23], and choose for normal hierarchy $m_{1}<m_{2}<m_{3}$ and for inverted hierarchy $m_{3}<m_{1}<m_{2}$. All active neutrino masses are taken to be positive.

One can easily see that for models II-IV one active neutrino is massless while the other two are degenerate. So, only the inverted hierarchy of active neutrino masses can be realized. As for the mixing angles in the active neutrino mass matrix, one finds for Model II: $\theta_{12}=\pi / 4, \theta_{23}=\pi / 2, \theta_{13}=-\arctan \left(h_{23} / h_{13}\right)$; for Model III: $\theta_{12}=\pi / 4, \theta_{23}=0, \theta_{13}=$ $-\arctan \left(h_{33} / h_{13}\right)$, and for Model IV $: \theta_{12}=\pi / 4, \theta_{13}=0, \theta_{23}=-\arctan \left(h_{33} / h_{23}\right)$, with a common choice of $\delta_{3}=0, \delta_{1}=\pi / 2$. Clearly, Models II and III cannot fit the data on neutrino oscillations, whereas Model IV is in fact quite close to the data, provided $h_{23} \simeq h_{33}$. This charge assignment corresponds to the so called $\left(L_{e}-L_{\mu}-L_{\tau}\right)$ symmetry [24, 25]. In the simplest forms of the breaking of this symmetry, the deviation of the solar mixing angle from its maximal value is too small, and this scheme is disfavoured by the data [26] (see, however, [27]).

We will choose, therefore, Model I as our starting point. The $U(1)_{L}$ global symmetry of this model is uniquely determined by the cosmological requirements formulated in the Introduction and by the data on neutrino oscillations. In this theory all three active neutrino masses are necessarily equal to zero and the mixing angles cannot be determined, so that both masses and angles are fixed by the breaking of the $U(1)_{L}$ symmetry. 


\section{SYMMETRY BREAKING AND ACTIVE NEUTRINO MASSES AND MIX- INGS}

The $U(1)_{L}$ symmetric theory constructed above contradicts experiment. Indeed, all active neutrino masses are zero. There is no candidate for dark matter particle simply because $\widetilde{N}_{2}$ and $\widetilde{N}_{3}$ are not stable and $\widetilde{N}_{1}$ is massless. The model does not contain any CP-violating phase and the baryon asymmetry cannot be produced. Therefore, the $U(1)_{L}$ symmetry cannot be exact. We will assume that this symmetry is slightly broken both by the Majorana mass terms and by the Yukawa interactions. So, we add to the symmetric Lagrangian the following symmetry-breaking terms

$$
L_{\text {breaking }}=\Delta L_{\text {mass }}+\Delta L_{\text {Yukawa }}
$$

where

$$
\Delta L_{\text {mass }}=-\frac{1}{2} \overline{\tilde{N}}^{c} \Delta M \widetilde{N}
$$

and

$$
\Delta L_{\text {Yukawa }}=-\bar{L} \Delta F \tilde{N} \Phi
$$

Explicitly,

$$
\Delta M=\left(\begin{array}{ccc}
m_{11} e^{i \alpha} & m_{12} & m_{13} \\
m_{12} & m_{22} e^{i \beta} & 0 \\
m_{13} & 0 & m_{33} e^{i \gamma}
\end{array}\right)
$$

Here all $m_{i j} \ll M$ can be taken to be real, $\alpha, \beta$, and $\gamma$ are three Majorana CP-violating phases.

The Yukawa part is taken to be

$$
\Delta F=\left(\begin{array}{lll}
h_{11} & 0 & h_{13} \\
h_{21} & 0 & h_{23} \\
h_{31} & 0 & h_{33}
\end{array}\right)
$$

where $h_{i 1} \ll h_{k 2}, h_{i 3} \ll h_{k 2}$ are in general complex, containing 3 physical CP-breaking phases.

We do not have at hand any deep theory guideline to choose this or that pattern of symmetry breaking. Therefore, for the choice of parameters, we will be using phenomenological considerations, related to dark matter and the baryon asymmetry of the Universe [1, 2]. To 
characterize the measure of the $U(1)_{L}$ symmetry breaking ${ }^{3}$, we introduce a small parameter $\epsilon=F_{3} / F \ll 1$, where $F_{i}^{2}=\left[h^{\dagger} h\right]_{i i}, h=F_{0}+\Delta F$, and $F_{2} \equiv F$.

Let us consider first the masses of sterile neutrinos in a theory with symmetry breaking. As for the lightest sterile neutrino, playing the role of dark matter particle, we get, expanding in powers of $m_{i j}$ :

$$
m_{s}=M_{1} \simeq\left(m_{11}-\frac{2 m_{12} m_{13} \cos \alpha}{M}\right)
$$

For the Majorana mass square difference of the heavier neutrinos, essential for baryogenesis ${ }^{4}$, we find

$$
\begin{array}{r}
M_{2}^{2}-M_{3}^{2} \simeq 2\left[4 m_{12} m_{13}\left(m_{12} m_{13}+M m_{33} \cos \gamma+M m_{22} \cos \beta\right)+\right. \\
\left.+M^{2}\left(m_{33}{ }^{2}+m_{22}{ }^{2}+2 m_{33} m_{22} \cos (\gamma+\beta)\right)\right]^{1 / 2}
\end{array}
$$

If, for example, all $m_{i j} \propto \epsilon^{n} M$, we obtain parametrically $\left|M_{2}-M_{3}\right| \sim M_{1}$. For a somewhat different breaking pattern: $m_{11} \propto m_{12} \propto m_{13} \propto \epsilon^{n} M, m_{33} \propto m_{22} \propto \epsilon^{2 n} M$ we obtain $M_{2}^{2}-M_{3}^{2} \sim M_{1}^{2}$.

The mass eigenstates $\left(N_{I}\right.$ without tilde) are related to $\widetilde{N}_{I}$ by the unitary transformation,

$$
\widetilde{N}=U_{R} N
$$

Different elements of the matrix $U_{R}$ are, parametrically:

$$
\begin{array}{r}
{\left[U_{R}\right]_{11} \simeq 1, \quad\left[U_{R}\right]_{1 i} \sim \frac{m_{1 i}}{M}, \quad\left[U_{R}\right]_{j 1} \sim \frac{m_{1 j}}{M}} \\
{\left[U_{R}\right]_{i j} \simeq \frac{e^{i \phi_{0}}}{\sqrt{2}}\left(\begin{array}{cc}
e^{i \phi_{1}} & e^{i \phi_{2}} \\
-e^{-i \phi_{2}} & e^{-i \phi_{1}}
\end{array}\right),}
\end{array}
$$

where $i, j=2,3$, and the phases $\phi_{k}$ are related to $\alpha, \beta, \gamma$ and $m_{k l}$ through some expressions, which we do not present here because of their complexity and of the fact that they will not be used in what follows. In is clear from eq. (17) that for small $\epsilon$ the heavy sterile neutrino mass eigenstates have almost identical Yukawa couplings to active neutrinos.

\footnotetext{
3 Of course, the symmetry breaking Yukawa coupling constants and masses are subject to renormalization and do depend on the normalization scale $\mu$. This dependence, however, is completely negligible for $\mu<M_{P l} \sim 10^{19} \mathrm{GeV}$ due to the smallness of Yukawa couplings in the $\nu \mathrm{MSM}$ and can be safely omitted.

4 We disregard here the contribution to the physical masses of heavier Majorana neutrinos, coming from the electroweak symmetry breaking, which is of the order of active neutrino masses, $\sim 0.05 \mathrm{eV}$.
} 
Now, let us turn to active neutrino masses. Their values can be found from the general see-saw formula:

$$
M_{\nu}=-\sum_{I} F^{*} \frac{v^{2}}{M_{I}} F^{\dagger} .
$$

The computation is simplified considerably by noting [1, 2, 28] that the coupling of the lightest sterile neutrino to active fermions must be very small to avoid the constrains coming from the over-closing of the Universe and from X-rays observations. This coupling contains three different contributions, the direct one, $\sim h_{i 1}$, and two induced, coming from the mixing defined in (17): $\sim h_{i 3} \frac{m_{13}}{M}$ and $\sim h_{i 2} \frac{m_{12}}{M}$. Unless a miraculous cancellation of different contributions takes place, all these terms must be smaller than $\mathcal{O}\left(10^{-12}\right)$. Thus, in the leading order we find that the smallest active neutrino mass is given by:

$$
m_{1}=\frac{F_{1}^{2} v^{2}}{m_{11}} .
$$

To find two other masses we expand eq. (18) with respect to small parameters $h_{i 1} / h_{k 2} \ll$ $1, \quad h_{i 3} / h_{k 2} \ll 1, m_{i j} \ll M$. In the leading approximation, the active neutrino mass matrix has a simple form

$$
\left[M_{\nu}\right]_{\alpha \beta}=\frac{v^{2}}{M}\left(\tilde{h}_{\beta 3} h_{\alpha 2}+\tilde{h}_{\alpha 3} h_{\beta 2}\right)
$$

where

$$
\tilde{h}_{\beta 3}=h_{\beta 3}-\frac{1}{2} \frac{m_{33}}{M} h_{\beta 2}
$$

The matrix (20) has one zero eigenvalue, corresponding to the lightest active neutrino, so one has to choose $m_{1}=0, m_{2}=m_{\text {sol }}$, and $m_{3}=m_{\text {atm }}$, for the case of normal hierarchy, or $m_{3}=0, m_{1}=m_{\text {atm }}-\Delta m / 2$, and $m_{2}=m_{\text {atm }}+\Delta m / 2$, for the case of inverted hierarchy (here $\left.\Delta m=m_{\mathrm{sol}}^{2} / m_{\mathrm{atm}}\right)$. The two non-zero eigenvalues of $M_{\nu} M_{\nu}^{\dagger}$ give for other active neutrino masses:

$$
m_{2,3}=\frac{v^{2}}{M}\left(F_{2} \tilde{F}_{3} \pm\left|h^{\dagger} \tilde{h}\right|_{23}\right)
$$

where $v=174 \mathrm{GeV}$ is the vacuum expectation value of the Higgs field, $\tilde{F}_{3}$ is constructed from $\tilde{h}_{\beta 3}$ in full analogy with $F_{3}$. Replacing $F_{2}$ by $F$ and $\tilde{F}_{3}$ by $\epsilon \tilde{F}$ we then have:

$$
\begin{aligned}
2 F^{2} v^{2} \epsilon / M & \simeq m_{\mathrm{atm}}[\text { normal hierarchy] } \\
F^{2} v^{2} \epsilon / M & \simeq m_{\mathrm{atm}}[\text { inverted hierarchy] }
\end{aligned}
$$

where $m_{\mathrm{atm}}=\sqrt{\Delta m_{\mathrm{atm}}^{2}} \simeq 0.05 \mathrm{eV}$ and $m_{\mathrm{sol}}=\sqrt{\Delta m_{\mathrm{sol}}^{2}} \simeq 0.01 \mathrm{eV}$. If the "vectors" $h_{i 2}$ and $\tilde{h}_{i 3}$ are almost parallel, the normal hierarchy of active neutrino masses is realized (this hap- 
pens automatically if $\left.\frac{m_{33}}{M} h_{\beta 2} \gg h_{\beta 3}\right)$; if they are almost orthogonal, the inverted hierarchy is achieved.

Let us now discuss whether this matrix can describe the observed neutrino mixings parametrized by $V$. Suppose that $M$ is fixed. Then the question is: Can eq. (20), con-

sidered as a set of six equations for six complex numbers $h_{i 2}, \tilde{h}_{i 3}$ always be solved? The answer to this question is always affirmative; moreover, the solution is not unique and can be parametrized by an arbitrary complex number $z$. This can be seen as follows: the matrix $M_{\nu}$ does not change if we replace $h_{i 2}$ by $z h_{i 2}$ and $\tilde{h}_{i 3}$ by $\tilde{h}_{i 3} / z$. Now, since we defined $\epsilon$ from $\tilde{F}_{3}=\epsilon F_{2}$ we have $\epsilon=|z|$. In other words, from the point of view of the data on neutrino oscillations, the measure of the breaking of the $U(1)_{L}$ symmetry cannot be determined and can be arbitrarily small. As for the well-known peculiarities of the active neutrino mixing matrix (smallness of the $\theta_{13}$ mixing angle and the maximal value of $\theta_{23}$ ), they can always be achieved with a certain choice of $h_{i j}$.

In the next section we will discuss the constraints on $\epsilon$ coming from the baryon asymmetry of the Universe and from dark matter.

\section{BARYON ASYMMETRY AND DARK MATTER}

We will require that the theory with global $U(1)_{L}$ symmetry, which is slightly broken, constructed in the previous section, gives the baryon asymmetry of the Universe. This will give us a lower bound on the parameter $\epsilon$, for the following reasons: first, if we decrease $\epsilon$, the Yukawa coupling $F$, required for explanation of the atmospheric neutrino mass scale (22), must increase (for $M$ fixed). If $F$ is too large, the sterile neutrinos $N_{2,3}$ will equilibrate well before the electroweak scale. In this case the production of baryon asymmetry is suppressed [2]. Second, in the limit $\epsilon \rightarrow 0$, the CP-violating effects in the neutrino sector go away. This fact, though, is less important than the first one.

To estimate these effects, consider the equilibration rate of $N_{1,2}$, which in our notation is given by [2, 15]

$$
\Gamma_{N} \simeq \frac{F^{2} T}{8} \sin \phi
$$

where $\sin \phi \simeq 0.02$. A deviation of the concentration of $N_{1,2}$ from the equilibrium one at 
temperature $T$ is then proportional to

$$
\delta n \sim \exp \left(-\frac{\Gamma_{N} M_{0}}{T^{2}}\right)
$$

where $M_{0} \simeq 7 \times 10^{17} \mathrm{GeV}$ appears in the time-temperature relation, $t=\frac{M_{0}}{2 T^{2}}$.

If

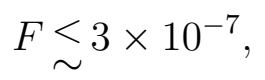

then $N_{2,3}$ do not equilibrate till they reach the temperature $T_{W} \simeq 130-190 \mathrm{GeV}$, corresponding to the freeze out of the sphaleron transitions [29], and the baryon asymmetry of the Universe is given by the general eqs. (22) and (29) of $[2]^{5}$ :

$$
\frac{n_{B}}{s} \sim 7 \times\left. 10^{-4} \operatorname{Tr} \Delta_{\mathrm{N}}\right|_{\mathrm{T}_{\mathrm{W}}}
$$

where the asymmetry in the sterile neutrino sector $\left.\Delta_{N}\right|_{T_{W}}$ is given by [2] :

$$
\left.\Delta_{N}\right|_{T_{W}} \sim a \frac{\pi^{\frac{3}{2}} \sin ^{3} \phi}{96 \cdot 3^{\frac{1}{3}} \Gamma\left(\frac{5}{6}\right)} \frac{\epsilon F^{6} M_{0}^{\frac{7}{3}}}{T_{W}\left(\Delta M_{32}^{2}\right)^{\frac{2}{3}}} .
$$

Here $a$ is a function of CP-violating phases that can be of the order of 1 . The asymmetry $\left.\Delta_{N}\right|_{T_{W}}$ is maximal and can be of the order of 1 provided the typical temperature of the lepton asymmetry generation, determined from the condition

$$
\frac{\left|M_{2}^{2}-M_{3}^{2}\right| M_{0}}{T^{3}} \sim 1
$$

coincides with the temperature at which sphaleron processes are switched off; this happens if $\left|M_{2}^{2}-M_{3}^{2}\right| \sim T_{W}^{3} / M_{0} \simeq(2 \mathrm{keV})^{2}$. Quite interestingly, a keV scale in the mass square differences of sterile neutrinos extremizes the baryon asymmetry.

If the lepton asymmetry production has a resonance character, a constraint on $F$ from above can be somewhat relaxed. Indeed, if $N_{2,3}$ are close to thermal equilibrium, the result (27) will be suppressed by $\delta n$ defined in (24), and $\delta n$ as small as $10^{-7}$ could still lead to the observed baryon asymmetry. This gives $F \lesssim 1.2 \times 10^{-6}$, which is about 4 times weaker than the requirement that reactions with $N_{2,3}$ be out of thermal equilibrium. This constraint, together with (22), leads to

$$
\epsilon \stackrel{\sim}{\sim} \times 10^{-4}(M / \mathrm{GeV})
$$

\footnotetext{
${ }^{5}$ Note that the matrix of the Yukawa couplings $F$ introduced in [2] was given in the Majorana mass eigenstate basis. It is equal to $\left(F_{0}+\Delta F\right) U_{R}$ in the notation of the present paper.
} 
for the normal hierarchy and to

$$
\epsilon \gtrsim 1.2 \times 10^{-3}(M / \mathrm{GeV})
$$

for the inverted hierarchy. If the value of $F$ from eq. (25) is taken, the values of $\epsilon$ have to be larger than those in (2930) by a factor of 16 .

Let us discuss now the constraints on the symmetry breaking pattern coming from dark matter. Since the coupling of dark matter sterile neutrinos to active neutrinos must be sufficiently suppressed, one should have $m_{12} \propto \epsilon^{p} M, m_{13} \propto \epsilon^{q} M$ and $h_{i 1} \propto \epsilon^{l} h_{i 2}$, with $p \geq 2, \quad q \geq 1$ and $l \geq 2$. A dark matter neutrino in the $\mathrm{keV}$ region would correspond to $m_{11} \propto \epsilon^{2} M$, for $M \sim 1 \mathrm{GeV}$. With this pattern of symmetry breaking an $\mathcal{O}(10) \mathrm{keV}$ dark matter neutrino could potentially be observed in astrophysical X-ray observations. However, with $\epsilon \sim 10^{-3}$ and $p \geq 3, \quad q \geq 2$ and $l \geq 3$, the decay rate $N_{1} \rightarrow \nu \gamma$ would be highly suppressed and observing a line in the X-ray background corresponding to radiative decays of an $\mathcal{O}(10) \mathrm{keV}$ sterile neutrino seems to be very difficult, if not impossible. As for sterile neutrino production, it could be in this case entirely related to interactions with the inflaton [3]].

\section{MASSES AND COUPLINGS OF SINGLET FERMIONS}

Let us discuss first possible values of masses of singlet fermions. As follows from Eqns. (29, 30), the smaller the mass $M$ of the singlet fermion, the more exact the $U(1)_{L}$ symmetry can be. So, the requirement of "naturalness" of the required sterile neutrino mass pattern favours light singlet fermions. However, very small masses are not allowed by the number of reasons. Indeed, the reducing of $M$ requires the simultaneous decrease of the Yukawa coupling $F$ (see eq. (22) ), which results in the decrease of the baryon asymmetry of the Universe, see eq. (27). This constraint, however, is rather weak and allows to have $M$ as small as few MeV. However, the lifetime of very light sterile neutrinos may be too large $\tau \gtrsim 10^{-2}$ s and thus spoil the predictions of BBN [30, 31].

To discuss the BBN constraints, let us consider first the $\nu \mathrm{MSM}$ without inflaton. In this theory singlet neutrinos decay trough the mixing with charged leptons and active neutrinos and the relevant limits can be extracted from [30, 31]. These limits can be contrasted with

experimental results of [32], what gives a lower bound on the mass of a singlet fermion 
$M \gtrsim 140 \mathrm{MeV}[33,34]$

Now, if light inflaton $\chi$ with mass $m_{\chi}$ is added to the theory, as in [3], singlet fermions acquire a new fast decay mode $N_{2,3} \rightarrow \nu \chi$, provided $M>m_{\chi}$, and the BBN constraints disappear if $M>$ few $\mathrm{MeV}$. In other words, singlet fermions, responsible for baryon asymmetry of the Universe, can be searched for at K2K, MiniBooNe and MINOS experiments in $K$ and even in $\pi$-meson decays. A discussion of different signatures of relatively light sterile neutrinos in a theory without inflaton can be found in [33].

Neutral fermions with masses $M>400 \mathrm{MeV}$ can be created in decays of $D$ and $B$-mesons or $\tau$-lepton. The possibilities for their experimental search will be discussed elsewhere [34] (existing experimental and BBN constraints for these masses are too weak, see [30, 31, 32, 33, 35]).

With a choice of $M$ of, say, $100 \mathrm{MeV}$ the measure of the breaking of the leptonic number symmetry can be as small as $\epsilon \sim 10^{-4}$. For larger masses the breaking of $U(1)_{L}$ must be stronger, see (29,301).

Now, let us turn to interactions of singlet fermions. The ratios of the Yukawa couplings $h_{i 2}$ can be expressed through the elements of the active neutrino mixing matrix. A particularly simple expression can be derived for the case $\theta_{13}=0, \theta_{23}=\pi / 4$, which is in agreement with the experimental data. For normal hierarchy four solutions are possible:

$$
\left|h_{12}\right|:\left|h_{22}\right|:\left|h_{32}\right| \approx \sqrt{\frac{m_{2}}{m_{3}}} \sin \theta_{12}|1 \pm x|: \frac{1}{\sqrt{2}}\left|1-x^{2}\right|: \frac{1}{\sqrt{2}}|1 \pm x|^{2}
$$

where $x=i e^{i\left(\delta_{1}-\delta_{2}-\delta_{3}\right)} \sqrt{\frac{m_{2}}{m_{3}}} \cos \theta_{12}$, and all combinations of signs are admitted. For a numerical estimate one can take [22] $\sin ^{2} \theta_{12} \simeq 0.3$, leading to $x \simeq 0.35 i e^{i\left(\delta_{1}-\delta_{2}-\delta_{3}\right)}$ and to $\left|h_{12}\right|^{2} /\left(\left|h_{22}\right|^{2}+\left|h_{32}\right|^{2}\right) \sim 0.05$. In other words, the coupling of the singlet fermion to the leptons of the first generation is suppressed, whereas the couplings to the second and third generations are close to each other.

For the case of inverted hierarchy two out of four solutions are almost degenerate and one has

$$
\left|h_{12}\right|:\left|h_{22}\right|:\left|h_{32}\right| \approx \sqrt{\frac{1+p}{1-p}}: \frac{1}{\sqrt{2}}: \frac{1}{\sqrt{2}}
$$

where $p= \pm \sin \delta_{1} \sin \left(2 \theta_{12}\right)$. Taking the same value of $\theta_{12}$ as before, we arrive at $\left|h_{12}\right|^{2} /\left(\left|h_{22}\right|^{2}+\left|h_{32}\right|^{2}\right) \sim(0.04-25)$, depending on the value of unknown CP-violating phase $\delta_{1}$. The couplings of $N_{2,3}$ to $\mu$ and $\tau$ generations are almost identical, but the coupling to electron and its neutrino can be enhanced or suppressed considerably. 
The corrections to relations (31,32) are of the order of $\mathcal{O}(\epsilon)$. These predictions can be easily translated into branching ratios of different decay modes and creation probabilities of heavy neutrinos and can be tested if these particles are found.

\section{CONCLUSIONS}

In this paper we constructed a variant of the $\nu \mathrm{MSM}$ in which the degeneracy of two singlet Majorana neutrinos and the lightness of the third one is a consequence of a lepton number symmetry, broken at a level of $\mathcal{O}\left(10^{-4}\right)$ in both the Yukawa and Majorana mass sectors. The resulting theory, as well as the more general $\nu \mathrm{MSM}$, explains dark matter, the baryon asymmetry of the Universe, and can be extended to have an inflaton, being consistent with the data on neutrino oscillations. In addition to predictions of a more general model in the active and sterile neutrino sectors, in the almost symmetric $\nu \mathrm{MSM}$ the couplings of two heavy neutrino mass eigenstates to active neutrinos are almost identical, and their flavour structure is fixed by observations of active neutrino oscillations. Moreover, the masses of these neutral leptons are likely to be relatively small, $M<1 \mathrm{GeV}$, which would allow their search in charmed, beauty, $K$ or $\pi$-mesons or $\tau$-lepton decays.

Besides that, the model predicts an outcome of a number of particle physics and astrophysics experiments. If the $\nu \mathrm{MSM}$ happens to be a correct effective theory up to the Planck scale, the LHC or other future accelerators will find no new particles, with the exception of the Higgs, unless dedicated experiments to search for heavier degenerate sterile neutrinos

(like those in [32, 35]), and, possibly, a light inflaton, are performed. No new sources of $\mathrm{CP}$-violation in the hadronic sector are foreseen. The direct and indirect searches of WIMP would give a negative result, but the dark matter particle could be discovered in X-rays.

The bottom-up approach used in this work cannot provide any fundamental reason for the existence of the $U(1)_{L}$ symmetry and explain the required pattern for its breaking. It would be interesting to see if the requisite symmetry and its breaking can come from some underlying dynamics at high energy scale. This problem, however, goes beyond the scope of the present paper.

Acknowledgements. This work was supported in part by the Swiss National Science Foundation. I thank Dmitry Gorbunov, Mikko Laine and Igor Tkachev for discussions and 
helpful remarks.

[1] T. Asaka, S. Blanchet and M. Shaposhnikov, Phys. Lett. B 631 (2005) 151.

[2] T. Asaka and M. Shaposhnikov, Phys. Lett. B 620 (2005) 17.

[3] M. Shaposhnikov and I. Tkachev, Phys. Lett. B 639 (2006) 414.

[4] A. Aguilar et al. [LSND Collaboration], Phys. Rev. D 64 (2001) 112007.

[5] [BooNE collaboration], http://www-boone.fnal.gov/

[6] S. Dodelson and L. M. Widrow, Phys. Rev. Lett. 72 (1994) 17.

[7] T. Asaka, M. Shaposhnikov and A. Kusenko, Phys. Lett. B 638 (2006) 401.

[8] A. Boyarsky, A. Neronov, O. Ruchayskiy and M. Shaposhnikov, Mon. Not. R. Astron. Soc. 370 (2006) 213.

[9] A. Boyarsky, A. Neronov, O. Ruchayskiy and M. Shaposhnikov, arXiv astro-ph/0603368.

[10] A. Boyarsky, A. Neronov, O. Ruchayskiy, M. Shaposhnikov and I. Tkachev, arXiv:astro-ph/0603660.

[11] S. Riemer-Sorensen, S. H. Hansen and K. Pedersen, Astrophys. J. 644 (2006) L33.

[12] U. Seljak, A. Makarov, P. McDonald and H. Trac, arXiv astro-ph/0602430.

[13] M. Viel, J. Lesgourgues, M. G. Haehnelt, S. Matarrese and A. Riotto, Phys. Rev. Lett. 97 (2006) 071301.

[14] C. R. Watson, J. F. Beacom, H. Yuksel and T. P. Walker, Phys. Rev. D 74 (2006) 033009.

[15] E. K. Akhmedov, V. A. Rubakov and A. Y. Smirnov, Phys. Rev. Lett. 81 (1998) 1359.

[16] V. A. Kuzmin, V. A. Rubakov and M. E. Shaposhnikov, Phys. Lett. B 155 (1985) 36.

[17] A. Kusenko and G. Segré, Phys. Lett. B 396 (1997) 197.

[18] P. L. Biermann and A. Kusenko, Phys. Rev. Lett. 96 (2006) 091301.

[19] J. Stasielak, P. L. Biermann and A. Kusenko, arXivastro-ph/0606435.

[20] T. Goerdt, B. Moore, J. I. Read, J. Stadel and M. Zemp, Mon. Not. Roy. Astron. Soc. 368 (2006) 1073, and references therein.

[21] P. Bode, J. P. Ostriker and N. Turok, Astrophys. J. 556 (2001) 93.

[22] A. Strumia and F. Vissani, Nucl. Phys. B 726 (2005) 294.

[23] B. Pontecorvo, Sov. Phys. JETP 6 (1957) 429; Sov. Phys. JETP 7 (1958) 172; Z. Maki, M. Nakagawa and S. Sakata, Prog. Theor. Phys. 28 (1962) 870. 
[24] S. T. Petcov, Phys. Lett. B 110 (1982) 245.

[25] R. Barbieri, L. J. Hall, D. R. Smith, A. Strumia and N. Weiner, JHEP 9812 (1998) 017.

[26] H. J. He, D. A. Dicus and J. N. Ng, Phys. Lett. B 536 (2002) 83.

[27] G. Altarelli and R. Franceschini, JHEP 0603 (2006) 047.

[28] A. Boyarsky, A. Neronov, O. Ruchayskiy and M. Shaposhnikov, Pis'ma v ZhETF 83 (2006) 165.

[29] Y. Burnier, M. Laine and M. Shaposhnikov, JCAP 0602 (2006) 007.

[30] A. D. Dolgov, S. H. Hansen, G. Raffelt and D. V. Semikoz, Nucl. Phys. B 590 (2000) 562.

[31] A. D. Dolgov, S. H. Hansen, G. Raffelt and D. V. Semikoz, Nucl. Phys. B 580 (2000) 331.

[32] G. Bernardi et al., Phys. Lett. B 203 (1988) 332.

[33] A. Kusenko, S. Pascoli and D. Semikoz, JHEP 0511 (2005) 028.

[34] D. Gorbunov and M. Shaposhnikov, in preparation.

[35] P. Astier et al. [NOMAD Collaboration], Phys. Lett. B 506 (2001) 27. 